\title{
Análise da demanda ambulatorial de fotodermatoses pré-malignas e malignas em uma universidade do sul catarinense
}

\section{Analysis of the demand of na ambulaatory of premalign and malign photodematoses in a south catharian university}

\author{
Débora Campos Pulido, Christine Horner, Fábio Almeida Morais, \\ Luiz Felipe de Oliveira Blanco
}

Como citar este artigo: PULIDO, DÉBORA C.; HORNER, CHRISTINE; MORAIS, FÁBIO A.; BLANCO, LUIZ F. O. Análise da demanda ambulatorial de fotodermatoses pré-malignas e malignas em uma universidade do sul catarinense. Revista Saúde (Sta. Maria). 2019; 45 (3).

\section{Autor correspondente:} Nome: Débora Campos Pulido

E-mail: deboracamposp@hotmail.com Telefone: (44) 99815000

Formação Profissional: Acadêmica do Curso de graduação em medicina da Universidade do Extremo Sul Catarinense Criciúma/SC.

Filiação Institucional: Universidade do Extremo Sul Catarinense

Endereço para correspondência: Av. Victor Meirelles

Bairro: Centro

Cidade: Criciúma

Estado: Santa Catarina

CEP: 88802-050

Data de Submissão:

13/10/2019

Data de aceite:

17/10/2019

Conflito de Interesse: Não há conflito de interesse

(cc) $\mathrm{BY}-\mathrm{NC}-\mathrm{ND}$

\section{RESUMO}

Objetivo: Conhecer a frequência das principais lesões de pele pré-cancerosas e malignas, em pacientes que receberam atendimento de dermatologia no ambulatório das clínicas integradas em uma universidade do sul catarinense. Metodologia: Foi realizado um estudo observacional, descritivo, retrospectivo, com coleta de dados secundários e abordagem quantitativa. Foram analisados 80 prontuários de pacientes de ambos os sexos atendidos entre 2017 e 2018. Resultados: Foi encontrada uma frequência de $62,5 \%$ de doenças pré-malignas e malignas de pele no sexo feminino, sendo que a idade média do início das lesões foi de $62,80 \pm 10,11$ anos. A maioria dos pacientes pesquisados eram aposentados $38,4 \%$, do lar $13,7 \%$ e autônomos $11 \%$, de forma que 59 pertenciam a raça branca, sendo que os demais prontuários não apresentaram tal informação. Quanto a hipótese diagnóstica, a ceratose actínica correspondeu a $59,4 \%$, seguida pelo carcinoma basocelular com $26,7 \%$, carcinoma espinocelular com $7,9 \%$ e melanoma com $5,9 \%$, sendo que a localização preferencial das lesões foi na face $52,2 \%$, com os membros representando $29,2 \%$ e o tronco $18,6 \%$. O tratamento iniciado foi na maior parte das vezes a exérese, em $47,4 \%$ dos indivíduos, seguida pela medicação tópica com 39,7\% e ambos ocorreram em 12,8\%. Conclusão: 0 perfil prevalente encontrado no ambulatório de dermatologia analisado foi de pacientes do sexo feminino (62,5\%), com uma idade média de início dos sintomas de $62,8 \pm 10,11$ anos, sendo $100 \%$ pertencentes à raça branca. A classe de hipótese diagnóstica mais encontrada foi a de ceratose actínica $(59,41 \%)$, e dentre as neoplasias malignas o carcinoma basocelular se destacou, com $26,7 \%$ dos casos, sendo que $52,2 \%$ das lesões foram encontradas em face, e em $47,4 \%$ a exérese foi o tratamento de eleição destas patologias. 0 motivo que explica a prevalência das lesões em pacientes mais idosos e de pele clara encontra-se no fato de que são necessários anos de exposição solar excessiva, e que esta etnia se apresenta mais suscetível aos danos provocados pelo sol, além de representarem a categoria predominante em nossa região. Da mesma forma, obtivemos a face como localização preferencial justamente por esse sítio permanecer maior tempo sem proteção solar. Devido as maiores informações sobre o tema e à popularização de instrumentos de proteção da radiação ultravioleta, acreditamos que, futuramente, ocorra queda no número de casos de fotodermatoses, acarretando, por conseguinte, diminuição da sua morbimortalidade.

PALAVRAS-CHAVE: Carcinoma; Ceratose actínica; Dermatologia; Luz solar.

\section{ABSTRACT}

Objective: Know the frequency of the main precancerous and malignant skin lesions in patients in dermatological care at the ambulatory of the Integrated Clinics in a university in southern Santa Catarina. Methodology: An observational, descriptive, retrospective study with secondary data collection and quantitative approach was performed. We analyzed 80 medical records of patients of both sexes attended between 2017 and 2018. Results: A frequency of $62.5 \%$ of malignant and premalignant skin diseases was 
found in females, with an average age of onset of lesions of $62.80 \pm 10.11$ years. Most of the patients surveyed were retired $38.4 \%$, housewives $13.7 \%$ and autonomous $11 \%$, so that $100 \%$ belonged to white breed, and the other records did not present such information. Regarding the diagnostic hypothesis, actinic keratosis corresponded to $59.4 \%$, followed by basal cell carcinoma with $26.7 \%$, squamous cell carcinoma with $7.9 \%$ and melanoma with $5.9 \%$, and the preferred location of the lesions was on the face 52.2 , with the limbs representing $29.2 \%$ and the upper body $18.6 \%$. The treatment initiated was mostly the exeresis in $47.4 \%$ of individuals, followed by topical medication with $39.7 \%$ and both occurred in $12.8 \%$. Conclusion: The prevalent profile found in the dermatologic ambulatory analyzed was female patients $(62.5 \%)$, with an average age at onset of symptoms of $62.8 \pm 10.11$ years, and $100 \%$ were white. The most commonly found diagnostic hypothesis was actinic keratosis (59.4\%), and among malignant neoplasms, basal cell carcinoma stood out, with $26.7 \%$ of the cases, and $52.2 \%$ of the lesions were found in the face, and in $47.4 \%$ the excision was the treatment of choice for these pathologies. The reason for the prevalence of lesions in older, fair-skinned patients is that years of excessive sun exposure are needed, and that this ethnicity is more susceptible to sun damage, and represents the category predominant in our region. Likewise, we obtained the face as a preferred location precisely because this site remains longer without sun protection. Due to the greater information on the subject and the popularization of instruments for the protection of ultraviolet radiation, we believe that in the future there will be a decrease in the number of photodermatosis cases, thus reducing its morbidity and mortality.

KEYWORDS: Carcinoma; Actinic keratosis; Dermatology; Sunlight.

\section{INTRODUÇÃO}

O tecido cutâneo representa o maior órgão do corpo humano, correspondendo a cerca de $15 \%$ do peso total do indivíduo. Ele envolve e delimita o organismo, preservando-o e relacionando-o com o ambiente externo. Assim como os demais órgãos, a pele é suscetível a fenômenos patogênicos, estabelecendo alterações micro e macroscópicas ${ }^{1}$.

As fotodermatoses são definidas como patologias cutâneas originadas ou motivadas pela exposição à luz solar, determinando modificações de caráter inflamatório ou degenerativo². Como importante representante de lesão prémaligna, a ceratose actínica (AK) é definida como um crescimento descontrolado de queratinócitos atípicos, possuindo capacidade de progressão para o carcinoma escamoso invasivo, por vezes desenvolvendo doença ameaçadora à vida ${ }^{3}$.

Atualmente, a neoplasia cutânea é a forma mais frequente e prevenível de todos os cânceres, podendo ser repartida em dois grandes grupos: melanoma e não melanoma. Os tipos não melanoma são mais habituais, porém menos severos, sendo representados pelos subtipos carcinoma basocelular (CBC) e carcinoma espinocelular (CEC). Entretanto, o tipo melanoma se caracteriza por ser menos comum, porém de caráter mais agressivo ${ }^{4}$.

A origem dessas patologias é multifatorial, estando a exposição solar em demasia e prolongada como fator de risco primordial ${ }^{5}$. Outros elementos associados a um maior risco são a predisposição genética, a existência de lesões préneoplásicas e imunossupressão sistêmica. Desenvolve-se mais frequentemente em adultos, principalmente na população idosa, e indivíduos de pele clara, consequentemente apresentando baixa incidência em negros, hispânicos e asiáticos ${ }^{4}$. 
Devido a região a ser estudada apresentar população majoritariamente de pele clara e predominância de idosos expostos a grande quantidade de raios UV, torna-se de fundamental importância conhecer tais afecções, para que seja realizado um diagnóstico precoce e um tratamento adequado para o tipo de lesão encontrada. 0 objetivo desse estudo foi conhecer a frequência das principais lesões de pele pré-cancerosas e malignas, em pacientes que receberam atendimento em um ambulatório de dermatologia.

\section{MATERIAIS E MÉTODOS}

Foi realizado um estudo observacional, descritivo, retrospectivo, com base nos prontuários dos pacientes acompanhados no ambulatório de dermatologia das Clínicas Integradas da UNESC, entre fevereiro de 2017 a dezembro de 2018.

A população deste estudo foi composta por 80 prontuários de pacientes atendidos no referido ambulatório e durante o período citado. Foram incluídos todos os pacientes cujos prontuários apresentaram suspeita de dermatose pré-cancerosa ou neoplasias cutâneas malignas, sendo excluídos aqueles que não tiveram o preenchimento de acordo com as necessidades exigidas pelo questionário, e nos quais não houveram suspeita clínica de lesão relacionada a foto exposição, seja ela de caráter maligno ou pré-maligno.

Para a coleta de dados, utilizaram-se informações de todos os prontuários dos pacientes atendidos, em que as variáveis solicitadas relacionavam-se ao sexo, idade atual, etnia, naturalidade, ocupação, data do início do acompanhamento médico, principal hipótese diagnóstica, uso prévio de medicação, uso contínuo de filtro solar, localização das lesões, tempo de início e descrição das mesmas, assim como os exames solicitados pelo médico a partir da suspeita clínica, tratamento iniciado, tempo para retorno do paciente ao serviço ambulatorial, presença prévia de patologia dermatológica e comorbidades.

Com base na ampla variedade de atividades laborais exercidas pelos pacientes analisados, eles foram classificados de acordo com as profissões mais prevalentes, incluindo 10 categorias: do lar, aposentado, trabalhador rural, professor, comerciante, costureira, motorista, autônomo, serviços domésticos e desempregado. Do mesmo modo, a variável naturalidade, por sua ampla gama de resultados apresentados, foi descrita com base em duas regiões predominantes, a saber: Criciúma e região e Rio Grande do Sul; as demais, devido menor prevalência, foram classificadas como "outras".

Acerca da localização das lesões encontradas em nosso estudo, destacamos, de uma forma geral, as três regiões do corpo com maior predominância de acometimento: face, tronco e membros. Devido a presença de múltiplos 
aspectos encontrados nas lesões, classificamos de acordo com as cinco características dominantes: hipercromia, eritematosa, perolada, enegrecida e hipocromia.

Como foi encontrada uma ampla variedade no que diz respeito a idade dos pacientes, foi utilizada a média para avaliação dessa variável. O tempo de início da lesão e o tempo para retorno foram expressos por meio da mediana. As variáveis qualitativas sexo, ano da primeira consulta, uso de medicação prévia para a lesão, uso de filtro solar, raça, hipótese diagnóstica, exames solicitados, histórico dermatológico e tratamento iniciado foram expressas por meio de frequência e porcentagem. Todos os resultados foram expressos por meio de tabelas.

Os dados coletados foram analisados com auxílio do software IBM Statistical Package for the Social Sciencies (SPSS) versão 21.0. As variáveis quantitativas foram expressas por meio de mediana e amplitude interquartil (com correção de Tukey) e por média e desvio padrão. As variáveis qualitativas foram expressas por meio de frequência e porcentagem.

Os testes estatísticos foram realizados com um nível de significância $a=0,05$ e, portanto, confiança de $95 \%$. A distribuição dos dados quanto à normalidade foi avaliada por meio da aplicação do teste de Shapiro-Wilk e Kolmogorov-Smirnov. A investigação da variabilidade das variáveis quantitativas entre as categorias das variáveis qualitativas foi investigada por meio da aplicação do teste de Levene.

A comparação da média das variáveis quantitativas entre as categorias das variáveis qualitativas dicotômicas foi realizada por meio da aplicação do teste $t$ de Student para amostras independentes quando observada distribuição Normal e U de Mann-Whitney quando a variável não seguiu esse tipo de distribuição.

A investigação da existência de associação entre as variáveis qualitativas foi realizada por meio da aplicação dos testes Qui-quadrado de Pearson e Exato de Fisher.

Este estudo foi aprovado pelo Comitê de Ética e Pesquisa (CEP) da Universidade do Extremo Sul Catarinense, sob o número de parecer 3.084.479, via Plataforma Brasil.

\section{RESULTADOS}

A população total estudada foi de 80 pacientes, dos quais 50 (62,5\%) eram do sexo feminino e 30 (37,5\%) do sexo masculino.

Com relação à idade de início dos sintomas, observou-se uma média de 62,80 anos $( \pm 10,11)$. Entre as profissões exercidas pelos pacientes, 28 (38,4\%) eram aposentados, $10(13,7 \%)$ do lar, $8(11 \%)$ autônomos, $7(9,6 \%)$ trabalhadores 
rurais, 7 (9,6\%) realizavam serviços domésticos, 5 (6,8\%) comerciantes, $3(4,1 \%)$ professores, $2(2,7 \%)$ costureiras, 2 $(2,7 \%)$ desempregados, $1(1,4 \%)$ motorista, e 7 não foram informados.

A respeito do ano da primeira consulta, 41 (51,3\%) ocorreram em 2017 e $39(48,7 \%)$ no ano de 2018.

No que se refere ao uso de medicação prévia para as lesões em questão, 38 (47,5\%) pacientes referiram ter aplicado algum fármaco. Do mesmo modo, apenas 19 (23,8\%) mencionaram o uso contínuo de protetor solar.

Quanto a etnia prevalente no estudo em questão, a branca foi a mais frequente, com 59 (100\%) indivíduos, sendo o restante não informado pelos prontuários. Por fim, a respeito da cidade de origem, Criciúma e região correspondeu a $71(89,9 \%)$ dos casos, seguido do Rio Grande do Sul com 6 (7,6\%), e demais localidades com 2 (2,5\%), sendo apenas 1 prontuário sem a definição de origem do paciente.

Tabela 1. Perfil dos pacientes atendidos no ambulatório de dermatologia das Clínicas Integradas da Universidade do Extremo Sul Catarinense nos anos de 2017 e 2018.

\begin{tabular}{lc}
\hline & Média \pm Desvio Padrão, $n(\%)$ \\
$n=80$
\end{tabular}

Sexo

$\begin{array}{ll}\text { Feminino } & 50(62,5) \\ \text { Masculino } & 30(37,5)\end{array}$

Ocupação

Aposentado $28(38,4)$

Do lar $\quad 10(13,7)$

Autônomo 8 (11)

Trabalhador rural $\quad 7(9,6)$

Serviços Domésticos $7(9,6)$

Comerciante $\quad 5(6,8)$

Professor $3(4,1)$

Costureira $2(2,7)$

Desempregado $2(2,7)$

Motorista $1(1,4)$

Não informado $\quad 7$

Ano da primeira consulta

2017

$41(51,3)$ 
Uso de medicação*

Uso de filtro solar

Raça

Branca

Não informado
$38(47,5)$

$19(23,8)$

$59(100,0)$

21

$71(89,9)$

$6(7,6)$

$2(2,5)$

Presença de comorbidades**

$61(77,2)$

Não informado 1

*Uso de medicação para lesão de pele (Fluoruracila, Ácido tricloroacético)

** Hipertensão arterial sistêmica, diabetes mellitus, osteoartrose, depressão

Fonte: Dados da pesquisa, 2019.

Com relação às classes de hipóteses diagnósticas pesquisadas, observou-se predomínio da ceratose actínica, com $59,4 \%$, seguida de carcinoma basocelular com $26,7 \%$, carcinoma espinocelular com 7,9\%, e o melanoma com $5,9 \%$. No que se refere a localização das lesões, a face foi a mais acometida $(52,2 \%)$, posteriormente membros $(29,2 \%)$, e tronco (18,6\%). Apenas um prontuário não informou esse dado.

No que concerne a descrição das lesões, a hipercromia foi a característica predominante $(54,7 \%)$, logo após, a eritematosa $(41,3 \%)$, a perolada $(17,3 \%)$, a enegrecida (4\%) e a hipocrômica (4\%). Foram encontrados cinco prontuários sem a descrição da lesão. 0 tempo médio de início do quadro clínico foi de 24 meses, sendo que em 36,3\% dos casos foi necessário algum exame complementar para elucidação diagnóstica.

A respeito do tratamento, a exérese foi a terapêutica mais utilizada $(47,4 \%)$, com o medicamento tópico correspondendo a 39,7\%, e ambos sendo necessários em 12,8\% dos casos. Apenas um prontuário não informou a intervenção realizada. Ademais, $77,2 \%$ dos pacientes apresentaram comorbidades, sendo que $33,8 \%$ referiu patologia dermatológica prévia. A média de tempo para retorno e acompanhamento da doença foi de 2,5 meses. 
Tabela 2. Características das lesões malignas e pré-malignas dos pacientes atendidos no ambulatório de dermatologia das Clínicas Integradas da Universidade do Extremo Sul Catarinense nos anos de 2017 e 2018.

\begin{tabular}{|c|c|}
\hline & $\begin{array}{c}\text { Mediana (AIQ), n (\%) } \\
n=80\end{array}$ \\
\hline \multicolumn{2}{|l|}{ Hipótese diagnóstica } \\
\hline Ceratose actínica & $60(59,41)$ \\
\hline Carcinoma basocelular & $27(26,73)$ \\
\hline Carcinoma espinocelular & $8(7,92)$ \\
\hline Melanoma & $6(5,94)$ \\
\hline \multicolumn{2}{|l|}{ Localização das lesões } \\
\hline Face & $59(52,21)$ \\
\hline Membros & $33(29,20)$ \\
\hline Tronco & $21(18,58)$ \\
\hline Não informado & 1 \\
\hline \multicolumn{2}{|l|}{ Descrição da lesão } \\
\hline Hipercrômica & $41(54,7)$ \\
\hline Eritematosa & $31(41,3)$ \\
\hline Perolada & $13(17,3)$ \\
\hline Enegrecida & $3(4,0)$ \\
\hline Hipocrômica & $3(4,0)$ \\
\hline Não informado & 5 \\
\hline Tempo de início da lesão (meses) & $24,0(12,0-60,0)$ \\
\hline Exames solicitados & $29(36,3)$ \\
\hline \multicolumn{2}{|l|}{ Tratamento iniciado } \\
\hline Exérese & $37(47,4)$ \\
\hline Medicamento tópico & $31(39,7)$ \\
\hline Ambos & $10(12,8)$ \\
\hline Não informado & 1 \\
\hline Tempo para retorno (meses) & $2,5(1,0-4,5)$ \\
\hline Histórico dermatológico & $27(33,8)$ \\
\hline
\end{tabular}

$\mathrm{AIQ}$ - Amplitude interquartil.

Fonte: Dados da pesquisa, 2019. 
Por fim, de acordo com a Tabela 3, quando associado o sexo com a hipótese diagnóstica, o feminino foi o mais acometido na ceratose actínica (60\%), no carcinoma basocelular (55,6\%), e no carcinoma espinocelular (62,5\%), sendo que o melanoma apresentou a mesma frequência de acometimento em ambos os sexos. Como descrito na Tabela 4, correlacionando a idade média do início do quadro clínico com a hipótese diagnóstica, constatou-se que na ceratose actínica a média de idade dos pacientes que apresentaram o diagnóstico foi de 64,02 ( $\pm 8,90)$, no carcinoma basocelular $62,74( \pm 10,20)$, no carcinoma espinocelular $67,13( \pm 10,43)$ e no melanoma $55,00( \pm 15,23)$.

Tabela 3. Analogia entre hipótese diagnóstica e sexo dos pacientes atendidos no ambulatório de dermatologia das Clínicas Integradas da Universidade do Extremo Sul Catarinense nos anos de 2017 e 2018.

\begin{tabular}{|c|c|c|c|}
\hline & \multicolumn{2}{|c|}{ Sexo, n (\%) } & \multirow[b]{2}{*}{ Valor-p } \\
\hline & $\begin{array}{c}\text { Feminino } \\
n=50\end{array}$ & $\begin{array}{c}\text { Masculino } \\
n=30\end{array}$ & \\
\hline \multicolumn{4}{|c|}{ Ceratose actínica } \\
\hline Sim & $36(60,0)$ & $24(40,0)$ & $0,424 ¥$ \\
\hline Não & $14(70,0)$ & $6(30,0)$ & \\
\hline \multicolumn{4}{|c|}{ Carcinoma basocelular } \\
\hline $\operatorname{Sim}$ & $15(55,6)$ & $12(34,4)$ & 0,8397 \\
\hline Não & $35(66,0)$ & $18(34,0)$ & \\
\hline \multicolumn{4}{|c|}{ Carcinoma espinocelular } \\
\hline Sim & $5(62,5)$ & $3(37,5)$ & $0,999 \dagger$ \\
\hline Não & $45(62,5)$ & $27(37,5)$ & \\
\hline \multicolumn{4}{|l|}{ Melanoma } \\
\hline Sim & $3(50,0)$ & $3(50,0)$ & $0,667 \dagger$ \\
\hline Não & $47(63,5)$ & $27(36,5)$ & \\
\hline
\end{tabular}

¥Valor obtido após aplicação do teste Qui-Quadrado de Pearson.

†Valor obtido após aplicação do teste Exato de Fisher.

Fonte: Dados da pesquisa, 2019. 
Tabela 4. Correlação entre hipóteses diagnósticas e idade média do início da lesão dos pacientes atendidos no ambulatório de dermatologia das Clínicas Integradas da Universidade do Extremo Sul Catarinense nos anos de 2017 e 2018.

\begin{tabular}{|c|c|c|c|c|}
\hline \multirow[t]{2}{*}{ Hipótese Diagnóstica } & \multirow[t]{2}{*}{$\mathrm{n}$} & \multicolumn{2}{|c|}{ Presença* } & \multirow[t]{2}{*}{ Valor $-p$} \\
\hline & & Sim & Não & \\
\hline Ceratose Actínica & 60 & $64,02 \pm 8,90$ & $59,15 \pm 12,66$ & $0,062 \uparrow$ \\
\hline Carcinoma Basocelular & 27 & $62,74 \pm 10,20$ & $62,83 \pm 10,16$ & $0,970 \dagger$ \\
\hline Carcinoma Espinocelular & 8 & $67,13 \pm 10,43$ & $62,32 \pm 10,03$ & $0,170+\dagger$ \\
\hline Melanoma & 6 & $55,00 \pm 15,23$ & $63,43 \pm 9,45$ & $0,049 \dagger$ \\
\hline
\end{tabular}

*Valores expressos por meio de média e desvio padrão.

†Valor obtido após aplicação do teste T.

†† Valor obtido após aplicação do U de Mann-Whitney.

Fonte: Dados da pesquisa, 2019.

\section{DISCUSSÃO}

No presente estudo foi possível observar uma prevalência de $62,5 \%$ do sexo feminino nos pacientes portadores das patologias analisadas. Neste sentido, uma análise retrospectiva em indivíduos do Pará no período de agosto a setembro de 2013 concluiu que, das 83 pessoas avaliadas, 75,9\% pertenciam ao sexo feminino ${ }^{2}$. Do mesmo modo, outro estudo realizado no Piauí no ano de 2010, também demonstrou predomínio de mulheres, com $63,7 \%{ }^{6}$. De forma oposta, outro trabalho realizado com 130 agricultores durante o ano de 2014, denotou que 60,8\% dos portadores da doença pertenciam ao sexo masculino ${ }^{7}$.

No que se refere à faixa etária de início dos sintomas, encontrou-se uma média de 62,80 anos $( \pm 10,11)$, o que coincide com um estudo realizado no interior de São Paulo, que avaliou as doenças de pele encontradas em trabalhadores rurais no ano de 2010, o qual encontrou uma maior incidência de lesões na faixa etária entre 60 e 80 anos, após análise de 143 pacientes, com 11 destes apresentando exposição solar danosa ${ }^{8}$. Igualmente, uma análise realizada com 130 trabalhadores rurais do Rio Grande do Sul, que avaliava a presença do câncer de pele, durante o ano de 2014, constatou uma faixa etária média de 55,67 $\pm 13,05$ anos $^{7}$.

Quanto à ocupação atual dos pacientes em questão, observamos que 38,4\% eram aposentados, seguido por $13,7 \%$ de trabalhadores do lar, $11 \%$ de autônomos e 9,6\% de agricultores. Nossos achados vão de encontro a uma pesquisa realizada na Dinamarca no ano de 2013 que relacionou a profissão com as lesões dermatológicas anteriormente citadas, demonstrando que dos 31 pacientes com diagnóstico, 25,8\% eram jardineiros e 16,1\% trabalhavam na construção civil $^{9}$. 
Dos 80 pacientes deste estudo, apenas $19(23,8 \%)$ referiram fazer uso contínuo de protetor solar, o que contrasta com uma pesquisa feito no interior do norte do Paraná em 2012, a qual denotou que 52,3\% dos 158 indivíduos estudados faziam uso diário de fotoprotetor ${ }^{10}$.

Em nossa análise, a raça branca foi a predominante, constituindo 100\% dos pacientes, dado que se assemelha ao apresentado em uma pesquisa realizada com trabalhadores rurais do Rio Grande do Sul no ano de 2014, na qual 100\% (130) também eram da raça branca, com fototipo Fitzpatrick $\mathrm{II}^{7}$, o mesmo ocorrendo em outra pesquisa realizada em um serviço de dermatologia de um hospital no Rio Grande do Sul no ano de 2010, em que $91,6 \%$ dos portadores de câncer de pele eram brancos ${ }^{11}$. Estes achados nos mostram que a raça branca, além de predominar na região sul do país, também favorece o desenvolvimento de doenças relacionadas a exposição solar.

As hipóteses diagnósticas em ordem decrescente de prevalência são a ceratose actínica com 59,41\%, 0 carcinoma basocelular com $26,73 \%$, o carcinoma espinocelular com 7,92\% e o melanoma com 5,94\%. Estes dados são corroborados por um estudo realizado em Manises, na Espanha, no ano de 2012, em que todos os habitantes da cidade foram analisados (25.956), sendo registrados 228 casos de ceratose actínica, 32 de carcinomas de pele não melanomas e 26 de melanoma ${ }^{12}$. Ademais, outra pesquisa realizada em um serviço de dermatologia de um hospital no Rio Grande do Sul no ano de 2010, que envolveu 1257 pacientes com diagnóstico de câncer de pele, demonstrou que 60,5\% dos casos eram compatíveis com carcinoma basocelular, 27,5\% com carcinoma espinocelular, e 8,4\% com melanoma ${ }^{13}$. A principal localização das lesões encontradas em nossa pesquisa foi a face, representando $52,21 \%$, o que vai ao encontro de outro estudo realizado na Dinamarca no ano de 2013, em que 24 (67\%) das lesões se localizavam na face e couro cabeludo $^{14}$, e de outro estudo realizado no norte do Paraná durante o ano de 2012, em que 79\% das lesões encontradas eram em face ${ }^{10}$.

Quanto a principal modalidade terapêutica utilizada, essa pesquisa demonstrou prevalência do tratamento cirúrgico, correspondendo a $47,4 \%$ dos casos, o mesmo sendo verificado no Rio Grande do Norte, em um estudo elaborado com 758 pacientes entre 2006 e 2013, em que 98,3\% dos pacientes efetuaram exérese da lesão ${ }^{13}$.

Adicionalmente, a respeito da presença de histórico dermatológico, 33,8\% dos pacientes analisados nesse estudo referiram algum tipo de lesão prévia de pele. Isso foi verificado em uma pesquisa feita no extremo sul do Rio Grande do Sul no ano de 2014, em que, dos 130 indivíduos avaliados, 7 deles (5,38\%) relataram quadro prévio de neoplasia maligna de pele ${ }^{7}$.

Quando correlacionamos a idade média do início das lesões com o diagnóstico, como demonstrado na tabela 4, observou-se que na ceratose actínica, dos 60 pacientes com a patologia, a média de idade foi de $64,02 \pm 8,90$ anos, fato também constatado pelo estudo realizado em Manises entre 2006 e 2012 em que a ceratose acometia principalmente indivíduos dos 51 aos 80 anos da idade ${ }^{12}$. Do mesmo modo, tal estudo foi ao encontro de nossa pesquisa no que se 
refere ao melanoma, o qual evidenciou predomínio entre 21 e 70 anos, o que condiz com a média encontrada por essa pesquisa, a qual foi de 55,00 $\pm 15,23$. Nos carcinomas basocelular e espinocelular a idade média foi de, respectivamente, $62,74 \pm 10,20$ e $67,13 \pm 10,43$, o que corroborou com o encontrado em uma pesquisa feita no Rio Grande do Norte nos anos de 2006 a 2013, em que 30 a 89 anos foi a faixa mais acometida no carcinoma basocelular e espinocelular, denotando uma importante semelhança entre ambos ${ }^{13}$.

Ademais, conforme verificado na tabela 3 a analogia entre a hipótese diagnóstica e o sexo prevalente, constatamos que o sexo feminino foi o mais encontrado em todos os casos, com exceção do melanoma, que obteve a mesma proporção entre mulheres e homens. Assim, na ceratose actínica as mulheres representaram $60 \%$ dos casos, da mesma forma que ocorreu em Tocantins durante uma análise em 2016, com elas correspondendo a 56,4\% dos indivíduos diagnosticados $^{15}$. Entre as neoplasias cutâneas, o carcinoma basocelular obteve 55,60\% de casos em mulheres e 0 espinocelular 62,5\%, assim como o estudo de Goiânia entre 1988 e 2009, com uma representatividade feminina de, respectivamente, $58,5 \%$ e $51,4 \%{ }^{16}$. Por fim, o melanoma, que em nossa análise apresentou igual predomínio entre homens e mulheres, neste mesmo estudo de Goiânia 63,5\% dos casos ocorreram no sexo feminino ${ }^{16}$, o que acreditamos se dever ao fato do baixo número de pacientes encontrados com tal diagnóstico em nosso ambulatório.

\section{CONSIDERAÇÕES FINAIS}

Constatou-se que a demanda prevalente das consultas dermatológicas com padrão pré-maligno e maligno no ambulatório analisado foi de pacientes do sexo feminino (62,5\%), com uma idade média de início dos sintomas de 62,8 \pm 10,11 anos, sendo 100\% pertencentes à raça branca. A classe de hipótese diagnóstica mais encontrada foi a de ceratose actínica $(59,4 \%)$, e dentre as neoplasias malignas o carcinoma basocelular se destacou, com $26,7 \%$ dos casos, sendo que $52,2 \%$ das lesões foram encontradas em face, e em $47,4 \%$ a exérese foi o tratamento de eleição destas patologias.

\section{REFERÊNCIAS}

1. Bardini G; Lourenço D, Fissmer MC. Avaliação do conhecimento e hábitos de pacientes dermatológicos em relação ao câncer de pele. Arquivos Catarinenses de Medicina. 2012 Nov; 2 (41): 56-63.

2. Araujo FC, Sousa BRMS, Leite GG, Freitas LC, Lemos ELC, Pires CAA. Avaliação dermatológica de agentes comunitários de saúde sujeitos à fotoexposição em região tropical do Brasil. Scientia Medica. 2016 Nov; 26 (4): 23897-2394. 
3. Schmitz L, Oster-Schmidt C, Stockfleth E. Nonmelanoma skin cancer - from actinic keratosis to cutaneous squamous cell carcinoma. Journal Of The German Society Of Dermatology. 2018 Jun; 26 (3): 1002-1013.

4. Zink BS. Câncer de pele: a importância do seu diagnóstico, tratamento e prevenção. Revista Hospital Universitário Pedro Ernesto. 2014 Ago; 13 (5): 76-83.

5. Polonini HC, Raposo NRB, Brandão MAF. Fotoprotetores Naturais como Instrumento de Ação Primária na Prevenção do Câncer De Pele. Aps. 2011 Abr; Juiz de Fora, 2 (14): 216-223.

6. Lages RB, Barbosa PB, Alemida IP, Lopes LRS, Filho LLL. Detecção precoce do câncer de pele: experiência de campanha de prevenção no Piauí-Brasil. Revista Brasileira em Promoção da Saúde. 2012 Jun; 25 (2): 221-227.

7. Cezar-Vaz, MR, Bonow CA, Piexak DR, Kowalczyk S, Vaz JC, Borges AM. Skin cancer in rural workers: nursing knowledge and intervention. Revista da Escola de Enfermagem da Usp. 2015 Ago; 49 (4): 0564-0571.

8. Hayashide JM, Minnicelli RS, Oliveira OAC, Sumita JM, Suzuki NM, Zambianco CA et al. Doenças de pele entre trabalhadores rurais expostos a radiação solar. Estudo integrado entre as áreas de Medicina do trabalho e Dermatologia: I. Revista Brasileira de Medicina do Trabalho. 2010 Jun; 8 (2): 221-227.

9. Caroe, TK, Ebbehøj NE, Christian H, Agner T. Recognized Occupational Skin Cancer in Denmark - Data From the Last Ten Years. Acta Dermato Venereologica. 2013 Jan; 93 (3): 369-371.

10. Garani R; Bertolini S. Câncer da pele em indivíduos acima de 50 anos de idade atendidos em um ambulatório de especialidades no norte do paraná. Revista Brasileira de Medicina. 2015 Fev; 38 (2): 1-60.

11. Silva AK, Santos FG, Budel F, Haeffner LSB, Farenzena GJ, Beber AAC. Câncer de pele: demanda de um serviço de dermatologia de um hospital terciário. Saúde (santa Maria). 2012 Nov; 38 (2): 55-64

12. Iranzo CC, Rubia-Orti JEDL, Castillo SS, Firmino-Canhoto J. Lesões cutâneas malignas e pré-malignas: conhecimentos, hábitos e campanhas de prevenção solar. Acta Paulista de Enfermagem. 2015 Fev; 28 (1): 2-6. 
13. Silva, TDC, Júnior GLS, Albuquerque RB, Oliveira ERRS, Batista KRF. Estudo retrospectivo de aspectos epidemiológicos, clínicos e histológicos na neoplasia de pele não melanoma. Revista Brasileira Cirurgia Cabeça e Pescoço. 2016 Marv; 45 (1): 1-16.

14. Caøroe, TK, Ebbehøj NE, Christian H, Agner T. Occupational skin cancer may be underreported. Danish Medical Journal. 2013 Mai; 60 (5): 24-46.

15. Silva WAR, Araújo HSO, Silva NMG, Noronha MPS, García-Zapata MTA, Pereira AL. Prevalência de ceratose actínica em idosos de uma região neotropical. Journal Health NPEPS. 2016; 1(2):208-217.

16. Pereira S, Curado MP, Ribeiro AMQ. Multiple skin neoplasms in subjects under 40 years of age in Goiania, Brazil. Revista de Saúde Pública. 2015 Nov; 49 (2): 49-64. 